\title{
Vortex Dipole in a Trapped Atomic Bose-Einstein Condensate
}

\author{
Qi Zhou, Hui Zhai \\ Center for Advanced Study, Tsinghua University, Beijing, China
}

(Dated: November 19, 2018)

\begin{abstract}
We calculate the angular momentum and energy of a vortex dipole in a trapped atomic BoseEinstein condensate. Fully analytic expressions are obtained. We apply the results to understand a novel phenomenon in the MIT group experiment [1], an excellent agreement is achieved, and further experimental investigation is proposed to confirm this vortex dipole mechanism. We then suggest an effective generation and detection of vortex dipole for experimental realization. Application of the sum rule to calculate collective mode frequency splitting is also discussed.
\end{abstract}

PACS number:

\section{INTRODUCTION}

The distinguished behavior of superfluid to carry angular momentum have attracted people's attention from long ago. It is well known that vortex excitation plays an important role when a superfluid responds to the external rotation. Nowadays, the vigorous development in cold atom physics has provided a new and more favorable arena to prove many theoretical predictions of rotating superfluid's behavior. It has been clearly demonstrated by many recent experiments that the vortex will be a stable excitation with quite long life-time when sufficient angular momentum is imparted, and the vortex lattice will be generated when even more angular momentum is brought to this system.

Besides the vortex itself, the vortex dipole, i.e., a bound vortex-antivortex pair, is also of particular interest. It is well known that the low-lying behavior of two-dimensional helium superfluid is dominated by vortex dipole, for the excitation energy of a vortex dipole is much smaller than a single vortex. With the increase of temperature, the vortex pairs will become unbounded and vortices will be free. This will lead to a phase transition known as Kosterlize-Thouless(KT) transition. Although no vortex-antivortex pair has yet been observed directly so far in atomic Bose-Einstein condensate(BEC), it is natural to presume that vortex dipole may play an important role when the imparted angular momentum is not large enough to support the vortex excitation. So it is worthwhile to study the properties of vortex dipole excitation in an atomic BEC.

The existence and importance of vortex dipole in an atomic BEC are also indicated by some recent experiments and numerical simulations. MIT group stirred the BEC locally with the stirring frequency which was too small to generate any surface mode, however, they observed a large number of vortices after stirring for some time. This suggested a local mechanism of generating vortex, say through vortex dipole excitation [1]. Recently some numerical calculation also indicated that stable vortex dipole could exist in a trapped atom BEC for a broad range of parameters 2]. All of these lead us to believe that cold atom physics will offer people opportunity to investigate this intricate vortex dipole excitation in su- perfluid. What is more, the characteristics of trapped atomic BEC will add much richer physics content to the properties of vortex dipoles, as well as to the experimental detection and production of vortex dipoles, which will be emphasized in this paper.

To proceed in this interesting and meaningful research field, we of course should first make clear the properties of a single vortex dipole in BEC, i.e., its angular momentum and energy. In section II, we will first deduce an exact result of its angular momentum and energy. It is not trivial to calculate the angular momentum and energy of vortex dipole in an inhomogeneous condensate. Here we use an integration method in complex coordinate to obtain fully analytical results, which will be described explicitly in this section and the appendix. We then apply our results to investigate MIT group experiment[1]. Through our calculations, we provide strong evidence to support the presence of vortex dipole during this kind of vortex nucleation process, and suggest further experiment to confirm this mechanism.

For experimental study the properties of vortex dipole in details, an effective way to generate and detect it is required. Although various methods have been widely adopted to produce vortices successfully, the formation of vortex dipole has not been systematically discussed. In section III, we will suggest that JILA's method [3], which generate vortex through a coherent spatial and temporal coupling between two-component BEC, could also be used to produce vortex dipole.

Measuring the density distribution could demonstrate the density depression in the neighborhood of the vortex center but have nothing to do with its phase. The same problem will be encountered in the case of vortex dipole. We could not distinguish which vortex is positive or negative only from the density image. What is more, the velocity field of a vortex dipole decreases quickly from the dipole center, and any closed path, which is a little far from the vortex dipole and includes both the vortex and antivortex, will be quite similar with the case that there is no singularity at all in this area. It is also not so effective to use interference method to reveal the phase information of vortex dipole as in vortex case 4]. However, experimentalists now could accurately measure the frequency splitting of the collective modes when the time reversal symmetry is broken due to the presence of vor- 
tices. This splitting will clearly distinguish vortex dipole from single vortex and vortex-free states. In theory, there are usually two ways to calculate the frequency of the collective mode in presence of vortices, one is perturbative calculation and the other is the sum rule approach. The results obtained from these two approaches are in good agreement with each other when the vortex is in the center of condensate. However, they are no more consistent when the vortex is off-center as pointed out in Ref. [5]. In section IV, we first use perturbation approximation to calculate the frequency splitting of the collective mode, and then we explain why the sum rule approach fails here. A brief conclusion and discussion will be given in the last section.

\section{PROPERTIES OF A VORTEX DIPOLE}

\section{A. Angular Momentum and Energy}

We hereafter consider a BEC confined in a pancake harmonic trap, which allows us to deal with a quasi-twodimensional system. The Gross-Pitaevski equation in a non-rotating coordinate frame is

$$
-\frac{\hbar^{2}}{2 m} \nabla^{2} \psi+V \psi+g|\psi|^{2} \psi=E \psi,
$$

where $V=\frac{1}{2} m \omega_{\perp}^{2} r^{2}$, and $g$ is the effective quasi-twodimensional interaction strength, obtained by integrating the usual three dimensional one $4 \pi \hbar^{2} a_{s c} / m$ over the $z$ direction. As we are considering the small angular momentum case, we could start with the Thomas-Fermi(T-F) approximation. So the density distribution of the ground state is well known as

$$
\rho(r)=\frac{\hbar \omega_{\perp}^{2} m}{2 g}\left(R^{2}-r^{2}\right),
$$

where $R$ is the T-F radius. Once a vortex dipole appears, in the region outside of vortex core we can write

$$
\psi=\sqrt{\rho(r)} e^{i \theta(r, \varphi)}
$$

with

$$
\theta(r, \varphi)=\Theta\left(\vec{r}-\vec{r}_{1}\right)-\Theta\left(\vec{r}-\vec{r}_{2}\right) .
$$

Here $\Theta(\vec{r})$ is the angle of $\vec{r}$ with respect to $x$ axis. $\vec{r}_{1}$ and $\vec{r}_{2}$ denote the positions of vortex and antivortex respectively. Eq.(3) follows from the assumption that the presence of vortices will not change the density distribution of the condensate but only introduce a phase, except inside the vortex core. This is true when the vortex core $\Lambda$ is small in the T-F region. With Eq. (3) we could calculate the angular momentum of the condensate as

$$
L=\hbar \int_{0}^{R} \rho r d r \int_{0}^{2 \pi} \frac{\theta(r, \varphi)}{\partial \varphi} d \varphi=\left.\hbar \int_{0}^{R} \rho r d r \theta(r, \varphi)\right|_{0} ^{2 \pi} \cdot(5)
$$

Only in the region $r_{1}<r<r_{2}, \theta$ will change $2 \pi$ after going along a circle. Thus,

$$
L=2 \pi \hbar \int_{r_{1}}^{r_{2}} \rho r d r .
$$

we notice that the energy of a vortex dipole increases with their distance, and the most efficient way to carry angular momentum is that $\vec{r}_{1}$ and $\vec{r}_{2}$ direct along the same radius, namely they are fully polarized, as are schematically shown in fig(11). So we could denote $r_{1}=D-d / 2, r_{2}=$ $D+d / 2$, therefore

$$
L=2 \pi \hbar \rho_{0} R^{2}\left(\frac{D d}{R^{2}}-\frac{D^{3}}{R^{3}} \frac{d}{R}-\frac{d^{3}}{4 R^{3}} \frac{D}{R}\right),
$$

where $\rho_{0}$ is $\hbar \omega_{\perp}^{2} m R^{2} /(2 g)$. Now we turn to the energy of

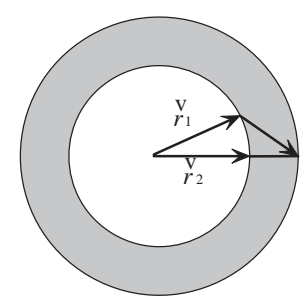

FIG. 1: Vortex dipole configuration: the grey torus expresses the effective part of the condensate which carries the angular momentum

the vortex dipole. In the T-F region, it could be written as

$$
E=\frac{\hbar^{2}}{2 m} \int \rho v^{2} d x d y
$$

where

$$
\vec{v}=\frac{\hat{z} \times\left(\vec{r}-\vec{r}_{1}\right)}{\left|\vec{r}-\vec{r}_{1}\right|^{2}}-\frac{\hat{z} \times\left(\vec{r}-\vec{r}_{2}\right)}{\left|\vec{r}-\vec{r}_{2}\right|^{2}} .
$$

Here we have neglected the presence of mirror dipole due to the edge effect, which would be a good approximation in a broad region except when the dipole is very close to the boundary of the condensate. For convenience, we do all the calculation in complex coordinates. We will use $z=x+i y$ and $\bar{z}=x-i y$, and then obtain

$$
E=\frac{\hbar^{2} \rho_{0}}{4 i m R^{2}} \int_{\Omega}\left(R^{2}-|z|^{2}\right) \frac{\left|z_{1}-z_{2}\right|^{2}}{\left|z-z_{1}\right|^{2}\left|z-z_{2}\right|^{2}} d \bar{z} \wedge d z .
$$

According to the Green formula

$$
\int_{\Omega} d \omega=\int_{\partial \Omega} \omega
$$

we could obtain the energy expression in the region $\Lambda \ll$ $d \ll R$

$$
E=\frac{2 \hbar^{2} \rho_{0}}{m}\left[1-\left(\frac{D}{R}\right)^{2}\right] \ln \frac{d}{\Lambda} .
$$


The detailed calculations are given in Appendix B. As the energy is dependent on $d$ logarithmically, the vortex and antivortex should bind up to each other closely, and we could neglect higher orders of $d / R$. Compared with the well known result for homogenous case, we also find that in Eq.(12) the homogenous density is replaced by local density at the position of vortex dipole. It can be easily understood because the amplitude of the velocity field of vortex dipole falls rapidly from the center of the vortex dipole, and most contribution to the energy of a dipole comes from the local velocity field.

\section{B. Interpretation of Experiment}

It is of long standing interest to investigate the intrinsic mechanism of vortex nucleation since the study of helium superfluid. In Ref. 1] dynamic nucleation of vortices in a trapped atomic BEC was studied experimentally. When the condensate was stirred by a laser with the beam waist comparable to the T-F radius, the condensate was globally rotated. In this case they found an enhanced vortex generation when the stirring frequency coincides that of surface excitations. It is indicated that vortex could be generated through surface excitation. However, when the condensate was stirred locally by a small beam, and the stirring frequency was far below any surface excitation frequency, they also found large number of vortices. This phenomenon suggested that the vortex can also be created locally in the bulk of the condensate, which seems to be in conflict with the topological argument, unless the vortex dipole was excited as an intermediate step. With the increase of angular momentum, the anti-vortices will finally be expelled out from the system and leave only the vortices in the condensate.

A remarkable feature of the local nucleation is that the vortex number produced by this local stirring is strongly dependent on the distance $l$ between stirring position and the center of condensate. The vortex number presents a maximum in the region where the ratio of $l$ to the condensate size $R$ is around 0.4. Hence, a question can be raised that whether we can understand the maximum from the properties of vortex dipole excitation.

Our answer to this question is, it is just in this region that the excitation energy of vortex dipole takes a minimum. In other word, the vortex dipole is easiest to be excited in this region. With Eq.(7) and Eq.(12) in hand, we could calculate the energy as a function of the position of vortex dipole $D$ for a given small value of angular momentum $L_{0}$. The result is

$$
\frac{E}{E_{0}}=\left(1-\left(\frac{D}{R}\right)^{2}\right) \ln \left[\frac{L_{0}}{2 \pi \hbar \rho_{0} R^{2}} \frac{R}{\Lambda(D)} \frac{1}{\frac{D}{R}-\left(\frac{D}{R}\right)^{3}}\right]
$$

where $E_{0}$ denoting $2 \hbar^{2} \rho_{0} / m$ is introduced as an energy unit. Here we should emphasize that the vortex core size
$\Lambda$ also depends on $D / R$ via

$$
\Lambda(D)=\frac{\Lambda_{0}}{\sqrt{1-\left(\frac{D}{R}\right)^{2}}} .
$$

This result is plotted in Fig(2). We could clearly see that the energy minimum occurs at $D / R$ around 0.4 . Our calculation thus provides a support to the vortex dipole mechanism.

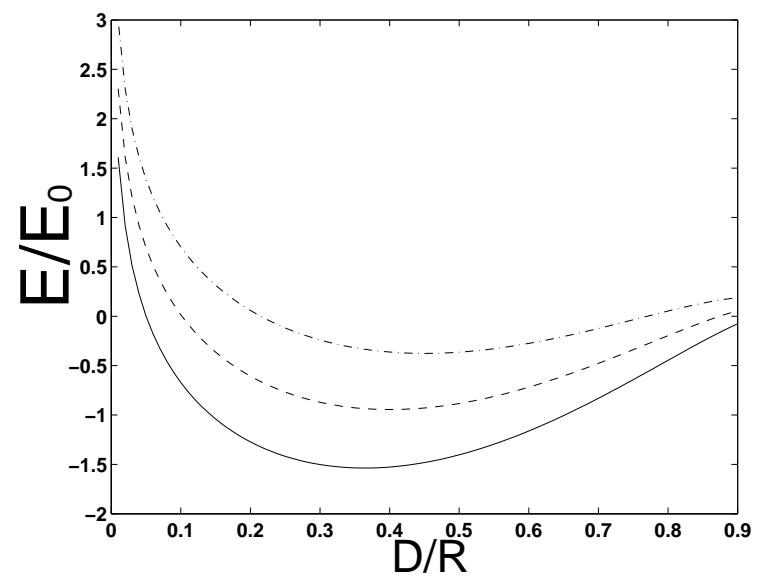

FIG. 2: Vortex dipole energy. Solid, dashed, and dash dot denote $T=0.05,0.1,0.2$ respectively, where $T=L_{0} / 2 \pi \hbar \rho_{0} R \Lambda_{0}$

To further confirm the vortex dipole mechanism, we investigate the properties of vortex dipole in an anharmonic trap

$$
V(r)=\frac{1}{2} m \omega_{\perp}^{2} r^{2}+\frac{1}{2} m k^{2} r^{4}
$$

The angular momentum and energy of the vortex dipole will become

$$
L=2 \pi \hbar \rho_{0} R^{2}\left[(1+A) \frac{D d}{R^{2}}-\frac{D^{3}}{R^{3}} \frac{d}{R}-A \frac{D^{5}}{R^{5}} \frac{d}{R}\right],
$$

and

$$
E=\frac{2 \hbar^{2} \rho_{0}}{m}\left[1-\left(\frac{D}{R}\right)^{2}+A\left(1-\left(\frac{D}{R}\right)^{4}\right)\right] \ln \frac{d}{\Lambda} .
$$

where $A=\frac{k^{2} R^{2}}{\omega_{\perp}^{2}}$. We find that with the increase of the quartic term, the minimum in the $E-D$ curve moves toward larger value of $D / R$ as shown in fig. (3) Hence we predict that the stirring position where the number of generated vortices reaches its maximum will also change correspondingly, if a similar experiment is performed in an anharmonic trap.

\section{VORTEX DIPOLE FORMATION}

Let us first briefly review the vortex formation by phase imprinting method[3], which used a laser 


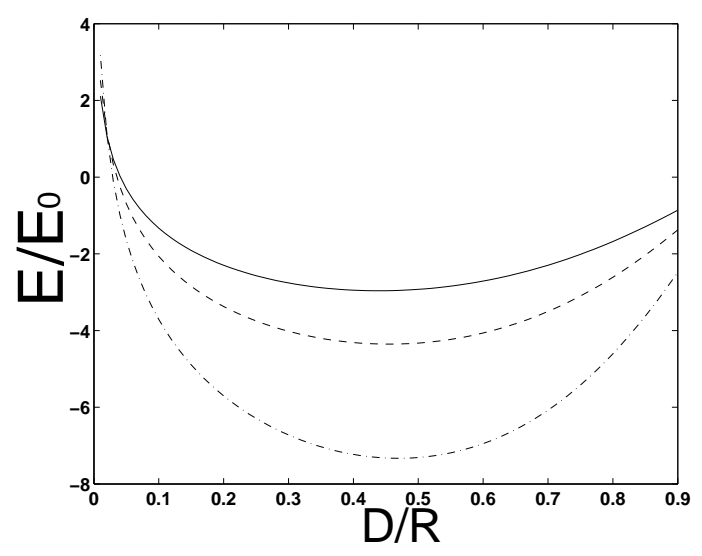

FIG. 3: Vortex dipole energy. Solid, dashed, and dash dot denote $A=0.5,1,2$ respectively, and $T$ is fixed at 0.05

to couple a two-component BEC. When we write the order parameter $\psi$ as $n^{1 / 2} e^{i \alpha} \chi$, where $\chi^{T}=$ $\left(\cos (\theta / 2) e^{-i \varphi / 2}, \sin (\theta / 2) e^{i \varphi / 2}\right)$, we could map the wave function to a pseudo-spin $\chi$ on a Bloch sphere. Under the optical coupling, the pseudo-spin will rotate with the effective Rabi oscillation frequency $\Omega_{\text {eff. }}$. In the experiment the laser was locally applied and moved around a circle with the frequency $\omega_{r}$, and the condensate in different points acquires a phase difference proportional to $\Omega_{\text {eff }} \Delta t$, where $\Delta t$ is the time interval of the laser passing through these two points. If $\omega_{r}=\Omega_{\mathrm{eff}}$, the phase difference between the two points is identical to $\Delta \theta$, and the phase configuration of a vortex is built up. Besides, if we only change the sign of $\Omega_{\text {eff }}$ and keep the circling direction of the laser unchanged, an anti-vortex will be produced.

Extending the above method to dipole generation is straightforward. From Fig.(4), we can see that the velocity field of a vortex dipole is clockwise in left plane and anti-clockwise in right plane. To produce a vortex dipole, the moving laser is required to change the sign of $\Omega_{\text {eff }}$ once it crosses the $y$ axis. What is more, the phase configuration of the vortex dipole could be produced exactly. $\Omega_{\text {eff }}$, the velocity of the moving laser $\vec{v}_{r}$ and the phase change gradient of the vortex dipole $\nabla \theta$ should satisfy the relationship

$$
\vec{v}_{r} \cdot \vec{\nabla} \theta=\Omega_{\mathrm{eff}}
$$

everywhere along the circle. $\theta$ denotes dipole phase expression Eq.(4). Once the phase along the circle is fixed, according to the uniqueness of solution to the equation $\nabla^{2} \theta=0$, the two phase singularities, as well as the phase elsewhere inside the circle will consequently be determined.

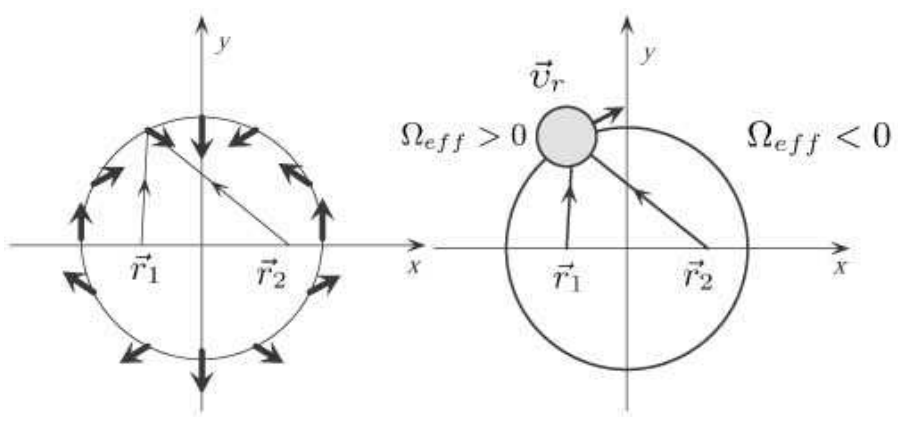

FIG. 4: Vortex dipole generation. Left: arrowhead denotes the velocity field of the vortex dipole along the circle. Right: $\vec{v}_{r}$ denotes the velocity of the moving laser

\section{VORTEX DIPOLE DETECTION}

When the ground state of the condensate possesses time reversal symmetry, the $\pm m$ collective modes will be degenerate. However, once the time reversal symmetry is broken due to the presence of vortex, the frequencies of the two modes will split. Zambelli and Stringari used the sum rule approach to obtain an analytical result of this splitting when a single vortex lies in the center of the condensate [6]. Svidzinsky and Fetter also obtained a consistent result by solving the the hydrodynamic equations with perturbational approximation 7]. They both found that the frequency splitting is proportional to the total angular momentum carried by the condensate. Later the frequency splitting when the vortex is off-center was also calculated pertubatively [] $[\underline{5}$. They found that the result obtained from the sum rule approach does not agree with that from the perturbative approach any more. In this section, we will first calculate the collective mode splitting in presence of a vortex dipole from the perturbation approximation, and we find the similar disagreement between perturbation approach and the sum rule approach will also occur here. We will briefly discuss the reason why the sum rule approach fails to meet the result of perturbative approach. We should point out that it is not the sum rule itself but the incorrect way we use it that leads to this failure.

\section{A. Frequency splitting induced by vortex dipole}

Substituting $\psi=\sqrt{\rho} e^{i \phi}$ into Eq.(1), we could obtain the well known hydrodynamic equations in the T-F region.

$$
\begin{aligned}
\frac{\partial \rho}{\partial t}+\vec{\nabla} \cdot(\rho \vec{v}) & =0 \\
M \frac{\partial \vec{v}}{\partial t}+\vec{\nabla}\left(V+g \rho-\mu+\frac{1}{2} M v^{2}\right) & =0
\end{aligned}
$$


where $\vec{v}=\vec{\nabla} \phi$. Expanding $\rho=\rho_{0}+\delta \rho$ and $\vec{v}=\vec{v}_{0}+\delta \vec{v}$ with

$$
\vec{v}_{0}=\vec{v}_{1}+\vec{v}_{2}=\frac{\hat{z} \times\left(\vec{r}-\vec{r}_{1}\right)}{\left|\vec{r}-\vec{r}_{1}\right|^{2}}-\frac{\hat{z} \times\left(\vec{r}-\vec{r}_{2}\right)}{\left|\vec{r}-\vec{r}_{2}\right|^{2}},
$$

and after linearization, we could obtain the equation for collective modes

$$
\left(\omega^{2}+\frac{g}{M} \vec{\nabla} \cdot \rho_{0} \vec{\nabla}\right) \delta \rho=-i \omega \vec{\nabla}\left(\vec{v}_{0} \delta \rho\right)+\frac{i g}{M \omega} \vec{\nabla} \cdot\left[\rho_{0} \vec{\nabla}\left(\vec{v}_{0} \cdot \vec{\nabla}\right) \delta \rho\right] .
$$

We notice that the left hand of Eq.(22) is just the equation of collective motion corresponding to a vortex-free ground state. And we also note that the effect of $v_{0}$ to the collective modes is always small, because $v_{0}$ decreases quickly from the vortex dipole center as $1 / r^{2}$ while $\delta \rho$ increases from the condensation center as $r^{m}$, their overlapping is always small except the position of the vortex dipole is nearby the boundary. So we could treat the right hand side as perturbation safely and obtain

$\omega^{2}-\omega_{0}^{2}=-i \omega_{0}\left\langle g\left|\vec{v}_{0} \cdot \vec{\nabla}\right| g\right\rangle+\frac{i g}{M \omega_{0}}\left\langle g\left|\vec{\nabla} \cdot \rho_{0} \vec{\nabla}\left(\vec{v}_{0} \cdot \vec{\nabla}\right)\right| g\right\rangle$,

where $\omega_{0}$ is the collective mode frequency in a vortex free case. Hereafter we consider the following surface mode

$$
|g\rangle=\sqrt{\frac{m+1}{\pi}} \frac{r^{m}}{R^{m+1}} e^{i \theta} .
$$

From the calculation presented in Appendix C, we could obtain the splitting between $+m$ mode and $-m$ mode as

$$
\omega_{+}-\omega_{-}=\frac{2(m+1)}{R^{2}}\left[\left(\frac{r_{2}}{R}\right)^{2 m-2}-\left(\frac{r_{1}}{R}\right)^{2 m-2}\right] .
$$

In experiments, from the optical absorption image the vortices positions $r_{1}$ and $r_{2}$ can be determined, and then by measuring the frequency splitting and comparing the obtained result with Eq.(25), one can distinguish a vortex dipole from two vortices with the same winding directions.

\section{B. Discussion on the Application of Sum Rule}

When we apply the formulism of Ref. [6] to calculate the frequency splitting of $\pm m$ modes, we can not obtain the same result as Eq.(25). To understand the reasons, let us first briefly remind ourself of the sum rules approach. The general formula of the sum rules for an arbitrary operator $\mathcal{F}$ can be easily proved as

$$
\begin{array}{r}
\sum_{n}\left(E_{n}-E_{k}\right)\left|\mathcal{F}_{n k}\right|^{2}=\frac{\left\langle k\left|\left[\mathcal{F}^{+},[H, \mathcal{F}]\right]\right| k\right\rangle}{2} \\
\sum_{n}\left(E_{n}-E_{k}\right)^{3}\left|\mathcal{F}_{n k}\right|^{2}=\frac{\left\langle k\left|\left[\left[\mathcal{F}^{+}, H\right],[H,[H, \mathcal{F}]]\right]\right| k\right\rangle}{2}
\end{array}
$$

If we choose $k=0$, and with a particular choice of the operator $\mathcal{F}$ which satisfies $\left|\mathcal{F}_{n 0}\right| \sim \delta_{n 0}$, we could obtain

$$
\hbar^{2} \omega_{n}^{2}=\frac{\left\langle 0\left|\left[\left[\mathcal{F}^{+}, H\right],[H,[H, \mathcal{F}]]\right]\right| 0\right\rangle}{\left\langle 0\left|\left[\mathcal{F}^{+},[H, \mathcal{F}]\right]\right| 0\right\rangle} .
$$

This is a powerful method because we can obtain the excitation energy directly from the property of the ground state without the explicit wavefunction of excitation state. However, the successful application of the sum rule approach relies on the proper choice of $\mathcal{F}$, which should excite the ground state to only one definitive excited state. When the system possesses some particular symmetry, we may easily find out a suitable $\mathcal{F}$. That's true when there is a central vortex, as rotation invariance is still present and the excited state should also have the form $e^{i m \phi}$. So we could easily select $\mathcal{F}$ as $(x \pm i y)^{2}$ to make use of the sum rule. However, the rotation invariance is broken in the case of vortex dipole. The choice of $\mathcal{F}$ still as $(x \pm i y)^{2}$ will excite several states, and Eq.(28) will not tenable. That's why the sum rule approach fails to agree with the perturbative result in Ref. [5].

\section{CONCLUSION}

In summary, by applying a convenient integration method in complex coordinate, we have obtained the fully analytic expressions of the angular momentum and energy of a vortex dipole in a trapped BEC. We also suggest an effective method to generate and detect the vortex dipole. Our work may provide a starting point for the research in the problem of vortex dipole excitation. There still remains many interesting fundamental issues in both theoretical and experimental physics, such as its dynamics, stability and detailed generation mechanism.

The precession of off-center vortex in a trapped condensate has been fully investigated both in experiment and theory. The mutual interaction between vortex and antivortex is presumed to bring a more nontrivial trajectory for vortex dipole. On the other hand, its dynamic behavior is closely related to its stability. In the dual electromagnetic picture [10], the positive and negative charge, to which the vortex dipole corresponds, will tend to annihilate each other via electromagnetic radiation, say phonon excitation in original picture. However, in a trapped condensate, there are two mechanism to stabilize the vortex dipole, one is the angular momentum conservation, and the other is the discrete spectrum of the the phonon in a finite system. Investigation of this interesting competition will reveal the underlying physics of the numerical simulation [2]. The fundamental mechanism of generating vortex dipole from a local stirring is even more complex, and it may resemble the birth of electron and positron from the vacuum polarization of the electromagnetic field.

Another important issue which should also arouse great attention is the complex phase diagram of rotating 
two-dimensional superfluid. When the angular momentum $\mathrm{L}$ equals zero, the critical temperature $T_{c}$ for $\mathrm{K}-\mathrm{T}$ transition is well understood. But when the superfluid carries a certain angular momentum, the dependence of $T_{c}$ to the angular momentum, as far as we are concerned, hasn't been clearly revealed. On the other hand, with the increase of angular momentum, the vortex dipoles will break into free vortices, and finally stable vortex excitation will become dominative. However, the explicit mechanism of the transition from vortex dipole excitation to stable vortex excitation is also still unknown. The interplay of the angular momentum and the thermal excitation will bring rich physics to the rotating twodimensional superfluid. Several works are proceeding.

Acknowledgements: The authors should like to thank Professor C. N. Yang for encouragement. And the authors would like to acknowledge for helpful discussions with Z.Y. Weng, L. Chang, R. Lü and X.L.Qi. QZ would like to thank Professor T.L.Ho and Professor Z.Y.Weng for their valuable support during his predicament excruciated by visa delay. QZ also thanks CASTU for providing a friendly environment to finish this work. This work is supported by National Natural Science Foundation of China ( Grant No. 10247002 )

\section{APPENDIX A: INTEGRATION IN COMPLEX COORDINATE AND GREEN FORMULA}

In this section we introduce our method used to do integration in a two-dimensional space. By denoting $z=$ $x+i y$ and $\bar{z}=x-i y$, we can turn the integration into the complex coordinate as

$$
\int_{\Omega} f(x, y) d x d y=-\frac{1}{2 i} \int_{\Omega} f(z, \bar{z}) d z \wedge d \bar{z},
$$

where $\Omega$ denotes the integration region, and the $\wedge$ operate fulfils $d x_{i} \wedge d x_{j}=\left(\delta_{i j}-1\right) d x_{j} \wedge d x_{i}$, here $x_{i}$ is the coordinate in real or complex space. Introducing a differential 1-form $\omega=g(z, \bar{z}) d z$ in which the function $g$ satisfies

$$
\frac{\partial g(z, \bar{z})}{\partial z}=f(z, \bar{z})
$$

we have $d \omega=f(z, \bar{z}) d z \wedge d \bar{z}$. According to the Green formula, we could change the above integration over $\Omega$ to over its boundary denoted as $\partial \Omega$ as

$$
\int_{\Omega} f(z, \bar{z}) d z \wedge d \bar{z}=\int_{\partial \Omega} g(z, \bar{z}) d \bar{z}
$$

This method is very useful when doing integration in a irregular region, because it turns a two-dimensional integration into a one-dimensional one, and result of the latter is easier to be obtained numerically or approximatively.

\section{APPENDIX B: VORTEX DIPOLE ENERGY}

To obtain the result of the integration Eq. (10) we first calculate

$$
\int_{\Omega} \frac{\left|z_{1}-z_{2}\right|^{2}}{\left|z-z_{1}\right|^{2}\left|z-z_{2}\right|^{2}} d \bar{z} \wedge d z .
$$

According to above method, it turns to

$$
\int_{\partial \Omega} \frac{z_{1}-z_{2}}{\left(z-z_{1}\right)\left(z-z_{2}\right)} \ln \frac{\bar{z}-z_{1}}{\bar{z}-z_{2}} d z
$$

We notice that not only does the integrand has two singularities $z_{1}$ and $z_{2}$, but also includes multi-value parts $\ln \left(\bar{z}-z_{1}\right)$ and $\ln \left(\bar{z}-z_{2}\right)$, so the integration in the boundary should be as the left side of Fig.(5).

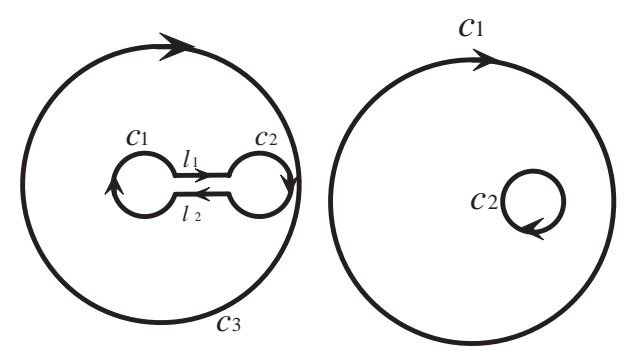

FIG. 5: The integration along the boundary in the complex coordinates.

In the condition that $d \gg \Lambda$, the integration along circle $C_{1}$ reads

$$
\begin{aligned}
& \int_{z-z_{1}=\Lambda e^{i \varphi}} \frac{z_{1}-z_{2}}{\left(z-z_{1}\right)\left(z-z_{2}\right)} \ln \frac{\bar{z}-z_{1}}{\bar{z}-z_{2}} d z \\
& \simeq \int_{0}^{2 \pi} \frac{-d}{\left(z-z_{1}\right)(-d)}\left[\ln \frac{\Lambda}{d}-i(\varphi+\pi)\right] i\left(z-z_{1}\right) d \varphi \\
& =-2 i \pi \ln \frac{\Lambda}{d}
\end{aligned}
$$

The similar result of integration along $C_{2}$ circle reads $2 i \pi \ln \frac{d}{\Lambda}$. We notice that the imaginary part of the logarithmic function in integrand in branch $l_{1}$ differs from that in $l_{2}$ by $2 \pi$, the integration along the two branches gives

$$
\int_{\Lambda}^{d-\Lambda} \frac{-d}{x(x-d)} 2 \pi d x=4 i \pi \ln \frac{d}{\Lambda} .
$$

The integration is more complex along circle $C_{3}$, we shall decompose Eq.(B2) as

$$
\sum_{i=1,2} \int \frac{\ln \left(z-r_{i}\right)}{\bar{z}-r_{i}} d \bar{z}-\sum_{i \neq j} \int \frac{\ln \left(z-r_{j}\right)}{\bar{z}-r_{i}} d \bar{z} .
$$

The general form of the integrand could be written as

$$
\frac{1}{\bar{z}}\left[1+\sum_{m=1}^{\infty}\left(\frac{z_{j}}{\bar{z}}\right)^{m}\right]\left[\ln z-\sum_{n=1}^{\infty}\left(\frac{z_{i}}{\bar{z}}\right)^{n}\right],
$$


then each of the four terms after decomposition is easier to be calculated. We obtain the integration along $C_{3}$ circle as

$$
2 i \pi\left[\ln \frac{\left(1-\frac{\left|z_{1}\right|^{2}}{R^{2}}\right)\left(1-\frac{\left|z_{2}\right|^{2}}{R^{2}}\right)}{\left(1-\frac{\left|z_{1} z_{2}\right|}{R^{2}}\right)^{2}}\right]
$$

The above value will vanish in the limit of $d / R \rightarrow 0$, thus could be neglected. Then we shall obtain

$$
\int_{\Omega} \frac{\left|z_{1}-z_{2}\right|^{2}}{\left|z-z_{1}\right|^{2}\left|z-z_{2}\right|^{2}} d \bar{z} \wedge d z=8 i \pi \ln \frac{d}{\Lambda} .
$$

Proceeding the similar steps, we could obtain

$$
\int_{\Omega} \frac{|z|^{2}\left|z_{1}-z_{2}\right|^{2}}{R^{2}\left|z-z_{1}\right|^{2}\left|z-z_{2}\right|^{2}} d \bar{z} \wedge d z=8 i \pi \frac{D^{2}}{R^{2}} \ln \frac{d}{\Lambda} .
$$

The energy of a vortex dipole will finally read

$$
E=\frac{2 \hbar^{2} \rho_{0}}{M}\left[1-\left(\frac{D}{R}\right)^{2}\right] \ln \frac{d}{\Lambda}
$$

\section{APPENDIX C: FREQUENCY SPLITTING}

We notice that the Eq. (23) is linear with respect to $v_{0}$, we could first calculate a single positive vortex case. That is

$$
v_{x}=-\frac{z^{\prime}-\bar{z}^{\prime}}{2 i z^{\prime} \bar{z}^{\prime}}
$$

and

$$
v_{y}=\frac{z^{\prime}+\bar{z}^{\prime}}{2 z^{\prime} \bar{z}^{\prime}}
$$

where $z^{\prime}=z-z_{1}, z_{1}$ denotes the location of vortex. Thus

$$
\left\langle g\left|\vec{v}_{0} \cdot \vec{\nabla}\right| g\right\rangle=\left\langle g\left|\frac{\partial_{z}}{\bar{z}-z z_{1}}-\frac{\partial_{\bar{z}}}{z-z_{1}}\right| g\right\rangle
$$

$$
\begin{aligned}
& =-\frac{m+1}{\pi R^{2 m+2}} \int_{\Omega} \frac{\bar{z}^{m}}{\bar{z}-z_{1}} \partial_{z} z^{m} d z \wedge d \bar{z} \\
& =-\frac{m+1}{\pi R^{2 m+2}} \int_{\partial \Omega} \frac{\bar{z}^{m} z^{m}}{\bar{z}-z_{1}} d \bar{z}
\end{aligned}
$$

The integration along the boundary is expressed as the right ride of Fig(5), and the result is easily to be obtained as

$$
\left\langle g\left|\vec{v}_{0} \cdot \vec{\nabla}\right| g\right\rangle=\frac{(m+1) i}{R^{2}}\left(1-\frac{\left|z_{1}\right|^{2 m}}{R^{2 m}}\right) .
$$

With the same process, we could also obtain $\langle g| \vec{\nabla}$. $\rho_{0} \vec{\nabla}\left(\vec{v}_{0} \cdot \vec{\nabla}\right)|g\rangle$ as

$$
-\frac{2 i m(m+1) \rho_{0}}{R^{4}}\left[1+\left(\frac{\left|z_{1}\right|}{R}\right)^{2 m}-2\left(\frac{\left|z_{1}\right|}{R}\right)^{2(m-1)}\right] .
$$

Recalling that $\omega_{0}=\omega_{\perp} \sqrt{m}$ and substituting the above expression back into Eq.(23), we will obtain

$$
\omega-\omega_{0}=\frac{m+1}{R^{2}}\left[1-\left(\frac{\left|z_{1}\right|}{R}\right)^{2(m-1)}\right] .
$$

The same result of Ref.([5]) is again obtained. As we have emphasized that Eq.(23) is linear with $v_{0}$, the above result of single vortex can be directly extended to vortex dipole, that is

$$
\omega-\omega_{0}=\frac{m+1}{R^{2}}\left[\left(\frac{\left|z_{2}\right|}{R}\right)^{2(m-1)}-\left(\frac{\left|z_{1}\right|}{R}\right)^{2(m-1)}\right],
$$

where $z_{2}$ is the position of anti-vortex. To calculate the frequency of $-m$ mode, we only need to change the sign of $v_{0}$ equivalently. So the frequency splitting of $\pm m$ mode is

$$
\omega_{+}-\omega_{-}=\frac{2(m+1)}{R^{2}}\left[\left(\frac{\left|z_{2}\right|}{R}\right)^{2(m-1)}-\left(\frac{\left|z_{1}\right|}{R}\right)^{2(m-1)}\right] .
$$

[1] C.Raman, J.R. Abo-Shaeer, J.M. Vogels, K. Xu and W. Ketterle, Phys. Rev. Lett 87, 210402 (2001)

[2] L. C. Crasovan, V. Vekslerchik, V. M. Péez-García, J. P. Torres, D. Mihalache, and L. Torner, Phys. Rev. A 68, 063609 (2003)

[3] M. R. Matthews, B. P. Anderson, P. C. Haljan, D.S. Hall, C.E. Wieman and E. A. Cornell, Phys. Rev. Lett 83, 2498 (1999)

[4] S. Inouye, S. Gupta, T. Rosenband, A. P. Chikkatur, A. Görlitz, T. L. Gustavson, A. E. Leanhardt, D. E. Pritchard, and W. Ketterle, Phys. Rev. Lett, 87, 080402 (2001)
[5] M. Guilleumas and R. Graham, Phys. Rev. A 64, 033607 (2001)

[6] F. Zembelli and S. Stringari, Phys. Rev. Lett 81, 1754 (1998)

[7] A. A. Svidzinsky and A. Fetter, Phys. Rev. A 58, 3168 (1998)

[8] A. L. Fetter and A. A. Svidzinsky, J. Phys: Condens. Matter 13, R135 (2001)

[9] V. Bretin, S. Stock, Y. Seurin, and J. Dalibard, Phys. Rev. Lett,92, 050403 (2004)

[10] E. Lundh and P. Ao, Phys. Rev. A,61, 063612 (2000) 